\title{
Role of two therapeutic interventions on balance in children with spastic diplegia and hemiparasis : A comparative study
}

Naglaa A. Zaky ${ }^{1 *}$, Nahed S. Thabet ${ }^{1}$ and Michael B. Banoub ${ }^{2}$

*Correspondence: naglaaomsa_m@hotmail.com

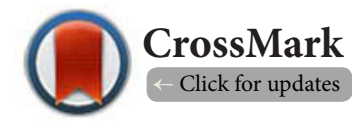

'Assistant Professor-Department of Physical Therapy for Pediatrics, Faculty of Physical Therapy, Cairo University, Egypt. ${ }^{2}$ Department of Physical Therapy for Cardiovascular-Pulmonary Disorders and Geriatrics, Faculty of Physical Therapy, Cairo University, Egypt.

\begin{abstract}
Background: Cerebral palsy $(\mathrm{CP})$ is the common movement disorder in children associated with life-long disability and multiple impairments. Their clinical manifestations vary among children and have a broad spectrum. Numerous physical therapy techniques are currently used for treatment as kinesio taping (KT) and electrical stimulation (ES) program training for anterior tibial group muscles.

Purpose: This study aimed to evaluate and compare the outcomes of using those techniques on balance in cerebral palsy children.

Methods: Sixty spastic CP children, their age ranged from four to six years were randomized and split equally between the diplegic CP group (15 received KT; eight boys, seven girls with mean \pm standard deviation $4.9 \pm 0.60$ years and 15 received ES; six boys, nine girls with mean \pm standard deviation $4.92 \pm 0.78$ years), and hemiparetic CP group (15 received KT; eight boys, seven girls with mean \pm standard deviation

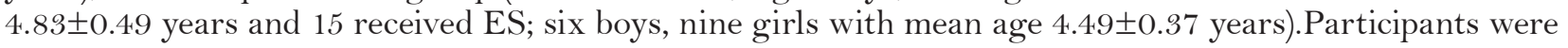
evaluated with the Biodex system before and after six months of treatment.
\end{abstract}

Results: Significant improvement was observed in the measured variables of the two groups when comparing their pre and post treatment mean values, significant improvement in comparing post treatment results in favor of KT in diplegic children and in favor of ES in hemiparetic children. Conclusion: Kinesio taping and electrical stimulation can be useful options to promote balance of children with $\mathrm{CP}$ with more pronounced effect of KT in diplegic type and ES in hemiparetic type.

Keywords: Cerebral palsy, Kinesio taping, Electrical stimulation, Balance

\section{Introduction}

Pediatric cerebral palsy (CP) is a non-progressive neurological disorder occurring in the immature brain, and is one of the most common causes of disability among children [1].

Cerebral cortical or sub-cortical insults occurring during the first year of life are clinically presented as a wide variety of disorders in $\mathrm{CP}$, and it is considered as the most prevalent cause of persisting motor function impairment [2]. The prevalence of CP was reported to be $2.5 \%$ per 1000 live births globally, and this abnormality varies with its involvement distribution [3]. Prevalence studies revealed that 3 to $4 \mathrm{CP}$ children were recorded per 1000 in the United States [4].

Abnormal muscle tone, postural reflexes and motor development are the primary characteristics of cerebral palsied children, as well as co-contraction, weakness or loss of movement control, defects in sensory integration, unsteady gait and impaired balance $[5,6]$.

Children with cerebral palsy have associated impairments in 
Zaky et al, Physical Therapy and Rehabilitation 2019,

http://www.hoajonline.com/journals/pdf/2055-2386-6-2.pdf

doi: $10.7243 / 2055-2386-6-2$

cognition, behavior, epilepsy and problems with perception, vision, and hearing, urinary incontinence, and constipation, which lead to restrictions in the ability to perform basic activities of daily living $[7,8]$.

A traditional classification of children with spastic CP includes topographical classification (hemiplegia, diplegia, and quadriplegia) or other classifications based on motor function as spastic and non-spastic (including athetoid, ataxic, and dystonic) $[9,10]$.

Based on the International Classification of Functioning system , CP affects the body structures e.g. limbs, body function e.g. intellectual function, activities (e.g. standing, walking, balance), and participation( e.g. sport). As a result of these deficits, some disabilities including impairments, limitation in function, and restriction in participation are subsequently occurring [11].

Children with $\mathrm{CP}$ have static and dynamic balance reactions that are less efficient when compared with those of typically developed children. As balance skills are an integral part of gross motor abilities, poor balance causes difficulty in performing functional tasks involved in activities of daily living [12].

The common standing pattern of children with spastic diplegia is characterized by ankles plantar flexion and hip medial rotation and adduction which considerably narrow the child's base of support (BOS). This narrowing of BOS leads to the accentuation of the impact of external perturbations, as it becomes more difficult to maintain the center of gravity inside a narrow base of support [13].

Children with CP have varying levels of deficits in balance, particularly in hemiplegic type, where one limb is predominantly affected. Asymmetrical behavior of the lower limbs is clinically manifested as gait asymmetry and it may be associated with a number of negative consequences which are challenges to control balance [14].

Rehabilitation of the patients with CP consists of a variety of commonly used therapeutic options including occupational and physical therapy [15]. Rehabilitation focuses on the restoration of muscle balance, proper alignment of joints and establishment of correct posture in the line of gravity. The success of any therapeutic program cannot be attained without achievement of these goals [16].

Kinesio tap (KT) is used for enhancing the activity, helps proprioception training as well as improves the muscle imbalance [17]. Although, the use KT with pediatric populations is not well studied, but studies showed that KT can further improve recovery in children with CP when applied in adjunct with traditional therapy interventions such as stretching, neurodevelopment therapy, practicing functional tasks [18].

Neuromuscular electrical stimulation (NMES) has been shown to be useful in the rehabilitation of neurological patients. It is used to strengthen the weak muscles and increase range of motion [19]. Although NMES is a therapeutic resource directed specifically to one muscle or muscle group, its results can be reflected in overall improvements in function $[20,21]$. Evaluation of balance by Biodex stability system provides a mode to assess levels of motor control that are mediated by lower extremity. It is considered a valid and reliable balance assessment. Biodex system is a unique device designed to stimulate joint mechanoreceptors and assess neuromuscular control by quantifying the ability to maintain dynamic postural stability $[22, \mathbf{2 3}]$.

The aim of this study is to investigate and compare the effects of kinesio tape and electrical stimulation on balance in diplegic and hemiparetic CP children.

\section{Materials and Methods \\ Study design \\ Randomized controlled clinical trial.}

\section{Subjects}

Spastic diplegic CP children and hemiparetic childern from both sexes, participated in the study, their ages ranged from 4 to 6 years. The study was held in the out-patient clinic of National Institute of Neuromotor System. Recruitment began after approval was obtained from the Ethics Committee of the Faculty of Physical Therapy, Cairo University.

Inclusion criteria for participation in the study included (a) A diagnosis of $\mathrm{CP}$ with Gross Motor Function Classification System (GMCFS) levels I and II [24]. (b) The degree of spasticity ranged from 1 and $1+$ according to the Modified Ashworth scale [25]. (c) Their height ranged between 100 and $113 \mathrm{~cm}$. (d) They could understand and follow orders. (e) They were able to walk independently with frequent falling.

Exclusion criteria included (a) A diagnosis of a genetic syndrome; (b) Children with any medical condition that would severely limit their participation in the study as vision or hearing loss, (c) Cardiac abnormalities or musculoskeletal disorders (d) Fixed deformities of lower limbs.

Initially, sample size estimation was done based on a Post -hoc test, a sample size of total 100 subjects would be required (GPower 3.0.9.2 program).

After fulfilling the eligibility criteria and the initial evaluation, the participants were distributed according to their diagnosis into a diplegic CP children (within it, 2 groups; KT and ES) and hemiparetic CP children (within it, 2 groups; KT and ES). Random process of assigning subjects to either the KT group or ES group was done by an assistant (physical therapist) who was not involved in any part of the study via using sealed opaque envelopes with code number for each patient in every group to help allocation of children in the groups. The distribution of subjects into the two groups at the end of the study was as follows; the diplegic CP group (15 in KT group ; eight boys, seven girls) and (15 in ES group; six boys nine girls) and hemiparetic CP group (15 in KT group; eight boys, seven girls) and (15 in ES group; six boys, nine girls).

\section{Instrumentation \\ Assessment instruments}

In baseline assessment for sample selection, GMFCS was used 
to assess severity of functional limitation/disability in children with cerebral palsy [24]. Modified Ashworth scale was also used for selection of subjects with 1 and 1+ degrees [25].

Biodex Balance System was used to assess balance, it consists of a movable balance platform, which provides up to 20 degrees of surface tilt in 360 degrees range. The system provides various degrees of surface instability which ranges from a completely firm surface; stability level (8) to a very unstable surface stability level (1) [26].

\section{Treatment instruments}

- Kinesio tape: It is latex-free with $100 \%$ cotton fibers, with an anti-allergic adhesive layer that allows for evaporation and quick drying. These properties make it resistant and wearable for a long period, and it is also water resistant. It is applied for the anterior tibial group muscles $[27,28]$.

- Electrical stimulator (Intelect advanced electro therapy system, model 2755CS, USA - Chattanooga group). It is used for the anterior tibial group muscles [29].

\section{Assessment procedures}

- Children were selected to be on level I and II based on GMCFS for Cerebral Palsy, children with motor problems classified on Level I, can generally walk without restrictions, but tend to be limited with regard to more advanced motor skills. Those classified on Level II, exhibit gait limitation in the outdoor environment [24].

Spasticity was assessed using the Modified Ashworth Scale. It measures the intensity of hypertonia, quantifying it as 0 (normal tonus) to 4 (rigidity) based on the degree of resistance the tested muscle offers to passive movement performed by the examiner. Assessment was done via testing tonus of the triceps surae during passive dosiflexion of the ankle with the knee flexed and extended.

Diplegic and hemiparetic CP children are selected if the degree of spasticity ranged from 1 to $1+$ according to this scale [25].

- Children' heights and weights were measured.

All parents had been informed about the study procedures. After signing a written consent form, instructions about evaluative procedures were explained for each child before the testing session to make sure that all children are familiarized with the equipment and the test. Evaluation for each child's balance by Biodex balance system was conducted in a warm and quiet room before and after six months of treatment. At first, certain parameters were fed to the device as child's age, height and weight (as measured using height scale SH-8024).

When the default testing protocol is performed ( 5 trials $x$ 20 seconds, 30 seconds rest) the reported Overall Stability Index can be compared to age related Predictive Values that were developed at the University of Southern Connecticut. The results of the five trials are collected and averaged by the software and displayed to the right of the age related Predictive Value. The Stability Index represents the variance of platform displacement in degrees from level in both the anterior/posterior and medial/lateral directions. A high number is indicative of poor neuromuscular control, which may increase the potential for orthopedic injury or falling.

The protocol used is as follows:

Test duration: 20 seconds, trials: 5 , rest between trials: 30 seconds, and stance: bilateral [30].

When assessment of children began, stability level was selected to be 5 (moderately stable) based on protocol of assessment in previous studies on CP children [31], The child was instructed to achieve a centered position on slightly unstable platform by shifting his feet position until keeping the cursor (which represents the center of the platform) centered on the screen while standing in a comfortable and upright position. Once centering was achieved and the cursor was adjusted in the center of the display target, instruction was given to the child to maintain his feet position till stabilizing the platform. This was followed by recording heels coordinates and feet angles from the platform. During postural stability testing, the child's ability to control the platform's angle of tilt is quantified as a variance from center. A large variance is indicative of poor neuromuscular control [32]. At the end of each test trial, a print out report was obtained. This report includes information regarding the tested variables which are; overall, anteroposterior and mediolateral stability indices.

\section{Treatment procedures}

Children in both groups received a selected physical therapy program during the six months of the study, for one-hour, three days a week on non-consecutive days according to their routine treatment, the session included the following items [33]:

(1) Standing with feet together while the therapist sitting behind and manually locking the child knees, and then slowly tilt him to each side, forward and backward, using mirror in front of child, (for $5 \mathrm{~min}$ ). (2) Step standing with therapist behind the child guiding him to shift his weight forward then backward alternately, using stepper, (for 5 min).(3) High step standing and try to keep balanced, using standing bar (for $5 \mathrm{~min}$ ). (4) Standing with manual locking of the knees then tries actively to stoop and recover (for $5 \mathrm{~min}$ ). (5) Equilibrium, righting and protective reactions training, using medical balls and tilting board, (for $5 \mathrm{~min}$ ). (6) Closed environment gait training in the form of forward, backward, and sideways walking between the parallel bars. Obstacles including rolls and wedges with different diameters and heights were put inside parallel bars, (for $5 \mathrm{~min}$ ). (7) Open environment gait training was conducted with obstacles as wooden blocks but without parallel bars, (for $5 \mathrm{~min}$ ). (8) Strengthening exercises for extensors muscles of the hip and knee,(for $10 \mathrm{~min}$ ). (9) Stretching exercises for tight muscles; hip flexors, hamstrings and calf muscles in lower limb and for wrist flexors, pronators and elbow flexors in upper limb (for $10 \mathrm{~min}$ ). Repetitions were from 2 to 3 times for each exercise according to the child's response. 


\section{Kinesio taping application}

-The strip is applied on the dorsal aspect of the foot then removed, the skin is observed for any reaction to the tape.

-If no reaction was detected, therapeutic application of the tape is sustained for successive 5 days, and then removed for 24 hours to allow skin preparation. Repetition of application and removal was conducted for successive six months [34].

- KT applications for the anterior tibial group muscles follows the protocol of Ragab et al., 2013 [35].

-Kinesio tex tape 2 inch in width $(5 \mathrm{~cm})$, in the form of two I strips was used.

-The first I strip was extended from the forefoot (dorsal aspect of metatarsals) to the anterior aspect of tibia just below the tibial tuberosity. The tape length was measured from the dorsal surface of the foot, while the ankle was dorsiflexed, to the anterior tibia below tibial tuberosity.

-Taping was initiated by first gently placing two inches of tap with paper -off tension on the dorsal aspect of the metatarsals. The foot was positioned in dorsiflexion and in mid position to fix the proximal anchor of the tape on the anterior tibia. Then the child was asked to plantar flex his ankle while therapist moved his both hands toward the middle of the tape to apply the remaining part of it.

- The second I strip tape was measured as twice the length of the lower leg, the tape backing in the center was splitted and the tape was placed on the plantar surface of the midfoot between calcaneous and metatarsal head with paper-off tension, then it was applied diagonally over the anterior ankle with increased tension on the lateral section to promote eversion. The tape was continued up the lower leg parallel to the first strip I.

-After tape application, each child was instructed not to make any activities for thirty minutes which was required for the tape to become fully activated. For diplegic group; KT was applied for both lower limbs while for hemiplegic group; it was applied for the affected lower limb.

\section{Electrical stimulation application}

-The child is positioned in supine position with a small pillow under his/her knee joint to maintain a slight flexed knee position.

- The electrodes were placed over anterior tibial group muscles, these area was cleaned with alcohol before application.

-The electrodes were fixed to the patient using fixing tape.

-One electrode was placed on the upper $1 / 3$ of anterior lateral surface of the lower leg just below the knee joint, the second electrode was placed distally on the lower leg (lower $1 / 3$ of anterior surface of the lower leg) to produce ankle dorsiflexion.

The stimulation parameters are: pulse frequency: 50 PPS (pulse per second), pulse duration $250 \mu \mathrm{s}$, cycle time: pulsed with 5 second stimulation and 10 seconds of rest, treatment time: 30 minutes. The intensity was set as high as the child can tolerate till a visible contraction was produced in the tibialis anterior muscle. During the intervention protocol, the intensity was increased to maintain sufficient contraction in order to generated ankle dorsiflexion. These parameters were chosen based on previous studies of electrical stimulation applications in cerebral palsy [36]. Sessions was held three days a week on non-consecutive days. For diplegic group; ES applied for both lower limbs while for hemiplegic group; it was applied for the affected lower limb.

\section{Data analysis}

The raw data of the study were analyzed using statistical package for the social sciences; SPSS Inc., Chicago, IL, USA, version 22. The dependent variables are the Overall Stability index, Anterior/posterior Stability index and Medial/lateral Stability index. Data are presented as mean \pm standard deviation for each measuring variables of the diplegic CP (KT and ES groups), and hemiparetic $\mathrm{CP}$ (KT and ES groups). Differences were assumed significant at $\mathrm{p}$-value $<0.05$. After data collection, Shapiro-Wilk test was used to detect normal distribution of data. For the parametric normally distributed data, paired t-test was used to detect differences within groups, and independent t-test was used to detect differences between both groups ( $K T$ and ES).

\section{Results}

For this study, 100 children were identified as potential participants ( 53 diplegic CP children and 47 hemiparetic CP children), twelve diplegic $C P$ children and thirteen hemiparetic CP children from screened subjects were excluded because they did not fulfill the inclusion criteria.75 subjects (diplegic $\mathrm{CP}$ and hemiparetic $(\mathrm{CP}$ ) were enrolled in the study. Of these children, 40 diplegic $C P$ and 35 hemiparetic CP. They were randomized into $\mathrm{KT}$ and $\mathrm{ES}$ groups, nine had got allergic reactions in KT groups, six had not yet completed the sessions and the final assessment. Data for thirty diplegic subjects and thirty hemiparetic subjects were available for final analysis ( 15 subjects in KT group and 15 subjects in ES group) (Figure 1).

As indicated by the independent t-test, there were no statistically significant differences $(P>0.05)$ between participants in both (KT and ES) diplegic CP groups, concerning age, height, and weight as shown in Table 1.

Table 1. Demographic characteristics of subjects in diplegic CP groups.

\begin{tabular}{lllll}
\hline Variable & $\begin{array}{l}\text { KT group, } \\
\mathbf{n}=\mathbf{1 5}\end{array}$ & $\begin{array}{l}\text { ES group } \\
\mathbf{n}=\mathbf{1 5} \\
\mathbf{X}^{-} \pm \mathbf{S D}\end{array}$ & $\begin{array}{l}\mathbf{X}^{-} \pm \mathbf{S D} \\
\text { T-value }\end{array}$ & P-value \\
\hline Age (years) & $4.9 \pm 0.60$ & $4.92 \pm 0.78$ & 0.079 & 0.938 \\
Height $(\mathrm{cm})$ & $107.86 \pm 3.41$ & $108.40 \pm 2.99$ & 0.454 & 0.653 \\
Weight $(\mathrm{kg})$ & $15.12 \pm 1.13$ & $15.21 \pm 1.01$ & 0.238 & 0.814 \\
$\begin{array}{l}\text { Gender } \\
\text { (boys/girls) }\end{array}$ & $8 / 7$ & $6 / 9$ & -- & -- \\
\hline
\end{tabular}

$\mathrm{X}^{-}$: Mean; SD: Standard deviation; P-value: Level of Significance, $\mathrm{P}>0.05$ : Non-Significant 


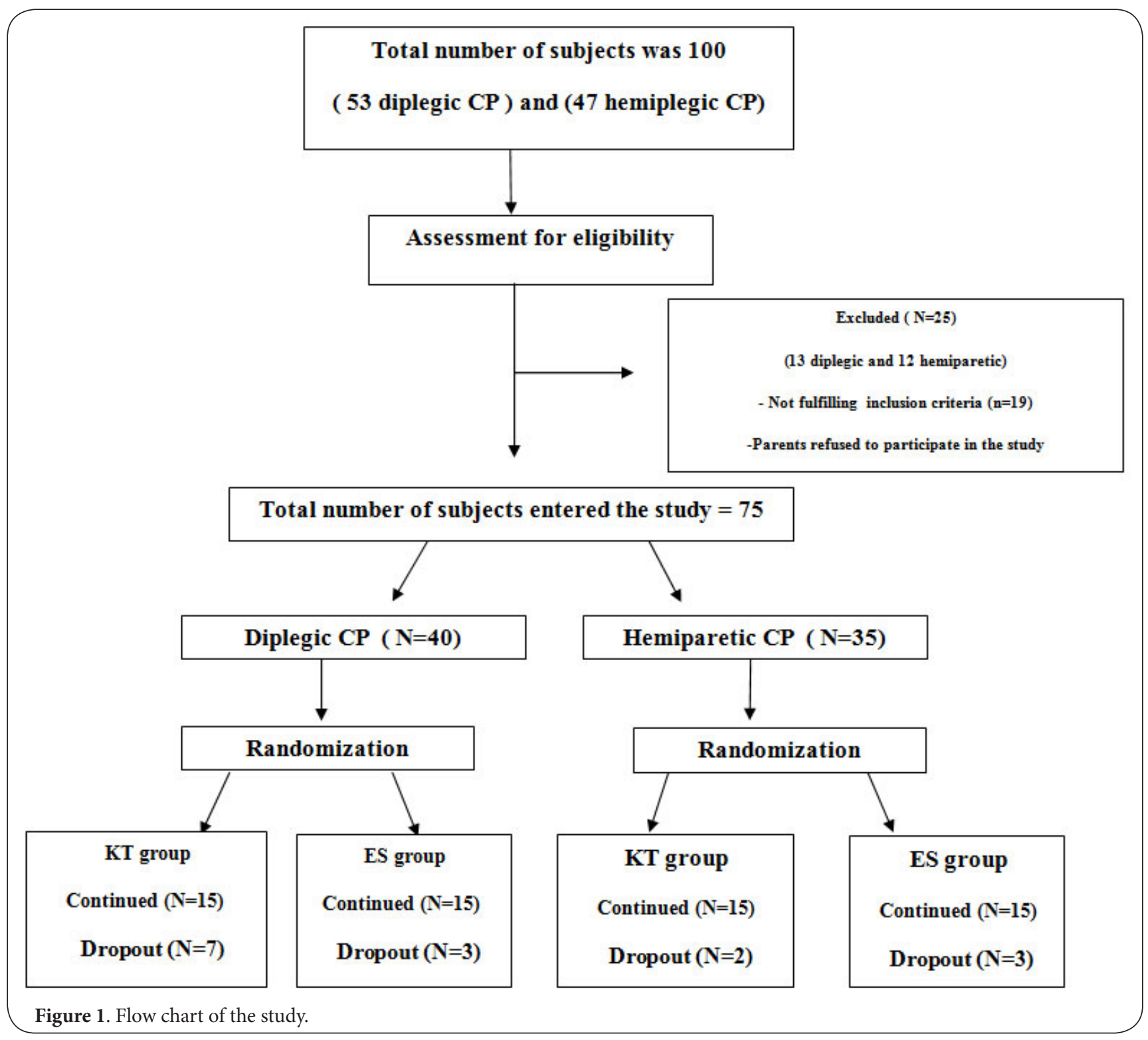

There were no statistically significant differences $(P>0.05)$ between participants in both (KT and ES) hemiparetic CP groups, concerning age, height, and weight as shown in Table 2.

The collected data from this study represent the statistical analysis of the stability indices including, overall stability index, antero-posterior (A/P) stability index and medio-lateral $(\mathrm{M} / \mathrm{L})$ stability index of the dynamic balance test, the variables were measured before and after six months of treatment for the two groups.

The obtained results in this study revealed no significant differences when comparing the pre-treatment mean values of the diplegic CP groups (KT and ES), As the mean values and SD of Overall SI were $(3.4887 \pm 0.1600)$, (3.4587 \pm 0.1688$)$ respectively. The mean values and SD of Antero-posterior SI were $(2.6380 \pm 0.2261)$ and $(2.6180 \pm 0.2261)$ respectively, The
Table 2. Demographic characteristics of subjects in hemiparetic CP groups.

\begin{tabular}{lllll}
\hline Variable & $\begin{array}{l}\text { KT group } \\
\mathbf{X}^{-} \pm \text {SD }\end{array}$ & $\begin{array}{l}\text { ES group } \\
\mathbf{X}^{-} \pm \mathbf{S D}\end{array}$ & T-value & P-value \\
\hline Age (years) & $4.83 \pm 0.49$ & $4.49 \pm 0.37$ & 0.209 & 0.836 \\
Height $(\mathrm{cm})$ & $107.73 \pm 3.23$ & $108.733 \pm 3.51$ & 0.810 & 0.425 \\
Weight $(\mathrm{kg})$ & $15.17 \pm 1.02$ & $15.21 \pm 1.14$ & 0.101 & 0.920 \\
$\begin{array}{l}\text { Gender } \\
\text { (boys/girls) }\end{array}$ & $8 / 7$ & $6 / 9$ & & \\
\hline
\end{tabular}

$\mathrm{X}^{-}$: Mean; SD: Standard deviation; P-value: Level of Significance, $\mathrm{P}>0.05$ : Non-Significant

mean values and SD of Medio-lateral SI were $(2.5567 \pm 0.1730)$ and $(2.4833 \pm 0.1688)$ respectively, where P-values were $(0.621)$, 
Zaky et al, Physical Therapy and Rehabilitation 2019,

$(0.810)$ and $(0.250)$ respectively. Also the obtained results revealed no significant differences when comparing the pre-treatment mean values of the hemiparetic $C P$ groups (KT and ES), As the mean values and SD of Overall SI were $(3.4680 \pm 0.1213),(3.4733 \pm 0.1486)$ respectively. The mean values and SD of Antero-posterior SI were (2.4800 \pm 0.2242$)$ and (2.4800 \pm 0.2242 ) respectively, The mean values and SD of Medio-lateral SI were $(2.4067 \pm 0.2120)$ and $(2.4267 \pm 0.1579)$ respectively where P-values were $(0.915),(1.00)$ and $(0.772)$ respectively.

As revealed from Table 3 , significant decrease was observed in the mean values of stability indices for the diplegic CP group at the end of treatment as compared with the corresponding mean values before treatment.

Also, Table 4, showed a significant reduction in the mean values of stability indices for the hemiparetic CP group at the end of treatment as compared with the corresponding mean values before treatment.

Significant improvement was observed in the measured variables when comparing post treatment results of the two groups in favor of KT in diplegic children and in favor of ES in hemiparetic children as shown in Tables $\mathbf{5}$ and $\mathbf{6}$.

\section{Discussion}

The purpose of this study was to investigate and compare the effects of kinesio tape and electrical stimulation on balance in diplegic and hemiparetic CP children.

It is well known that the children with cerebral palsy have varying levels of deficits in balance and postural control [37].

Studies have shown that GMFCS is recommended to use with cerebral palsy children to assess the growth motor abilities limitation for its high reliability and validity $[24,38]$.

Balance control is essential for competence in the perfor-

Table 3. Pre and post-treatment mean values of the stability indices for the diplegic CP groups.

\begin{tabular}{lllllll}
\hline Variable & Groups & $\begin{array}{l}\text { Pre treatment } \\
\text { Mean } \pm \text { SD }\end{array}$ & $\begin{array}{l}\text { Post treatment } \\
\text { Mean } \pm \text { SD }\end{array}$ & $\begin{array}{l}\text { Percent of } \\
\text { change\% }\end{array}$ & T value & P value \\
\hline Over all SI & KT group & $3.4887 \pm 0.1600$ & $2.9267 \pm 0.1989$ & 16.109 & 24.916 & $0.000^{*}$ \\
& ES group & $3.4587 \pm 0.1688$ & $3.1133 \pm 0.1805$ & 9.986 & 18.108 & $0.000^{*}$ \\
\hline Antero-posterior SI & KT group & $2.6380 \pm 0.2261$ & $2.0533 \pm 0.2021$ & 22.16 & 26.403 & $0.000^{*}$ \\
& ES group & $2.6180 \pm 0.2261$ & $2.3233 \pm 0.2069$ & 11.256 & 14.753 & $0.000^{*}$ \\
\hline Medio-lateral SI & KT group & $2.5567 \pm 0.1730$ & $1.9700 \pm 0.1567$ & 22.947 & 22.534 & $0.000^{*}$ \\
& ES group & $2.4833 \pm 0.1688$ & $2.1233 \pm 0.1944$ & 14.496 & 16.039 & $0.000^{*}$ \\
\hline
\end{tabular}

SI: Stability index. ; $\mathrm{X}^{-}$: Mean; SD: Standard deviation; P-value: Level of Significance, ${ }^{\star} \mathrm{p}<0.05$ :Significant

Table 4. Pre and post-treatment mean values of the stability indices for the hemiparetic CP groups.

\begin{tabular}{lllllll}
\hline Variable & Groups & $\begin{array}{l}\text { Pre treatment } \\
\text { Mean } \pm \text { SD }\end{array}$ & $\begin{array}{l}\text { Post treatment } \\
\text { Mean } \pm \text { SD }\end{array}$ & $\begin{array}{l}\text { Percent of } \\
\text { change\% }\end{array}$ & T value & P value \\
\hline Over all SI & KT group & $3.4680 \pm 0.1213$ & $3.1333 \pm 0.1175$ & 9.651 & 15.084 & $0.000^{*}$ \\
& ES group & $3.4733 \pm 0.1486$ & $2.9333 \pm 0.1676$ & 15.547 & 28.386 & $0.000^{*}$ \\
\hline Antero-posterior SI & KT group & $2.4800 \pm 0.2242$ & $2.1800 \pm 0.2274$ & 12.096 & 12.550 & $0.000^{*}$ \\
& ES group & $2.4800 \pm 0.2242$ & $1.9267 \pm 0.2219$ & 22.310 & 33.488 & $0.000^{*}$ \\
\hline Medio-lateral SI & KT group & $2.4067 \pm 0.2120$ & $2.0733 \pm 0.1944$ & 13.85 & 14.349 & $0.000^{*}$ \\
& ES group & $2.4267 \pm 0.1579$ & $1.8867 \pm 0.1684$ & 22.252 & 25.256 & $0.000^{*}$ \\
\hline
\end{tabular}

SI: Stability index. ; $\mathrm{X}^{-}$: Mean; SD: Standard deviation; P-value: Level of Significance, ${ }^{\star} \mathrm{p}<0.05$ : Significant

Table 5. Post-treatment mean values of the stability indices for the diplegic CP groups.

\begin{tabular}{lllll}
\hline Variable & Groups & Mean \pm SD & T value & P value \\
\hline Over all SI & KT group & $2.9267 \pm 0.1989$ & 2.691 & $0.012^{\star}$ \\
& ES group & $3.1133 \pm 0.18050$ & & \\
\hline Antero-posterior SI & KT group & $2.0533 \pm 0.2021$ & 3.615 & $0.001^{\star}$ \\
& ES group & $2.3233 \pm 0.2069$ & & \\
\hline Medio-lateral SI & KT group & $1.9700 \pm 0.1567$ & 2.378 & $0.024^{*}$ \\
& ES group & $2.1233 \pm 0.1944$ & & \\
\hline
\end{tabular}

SI: Stability index. ; $\mathrm{X}^{-}$: Mean; SD: Standard deviation;

P-value: Level of Significance, ${ }^{*} \mathrm{p}<0.05$ :Significant 
Zaky et al, Physical Therapy and Rehabilitation 2019,

Table 6. Post-treatment mean values of the stability indices for the hemiparetic CP groups.

\begin{tabular}{lllll}
\hline Variable & Groups & Mean \pm SD & T value & P value \\
\hline Over all SI & KT group & $3.1333 \pm 0.1175$ & 3.784 & $0.001^{*}$ \\
& ES group & $2.9333 \pm 0.1676$ & & \\
\hline Antero-posterior SI & KT group & $2.1800 \pm 0.2274$ & 3.088 & $0.005^{\star}$ \\
& ES group & $1.9267 \pm 0.2219$ & & \\
\hline Medio-lateral SI & KT group & $2.0733 \pm 0.1944$ & 2.810 & $0.009^{*}$ \\
& ES group & $1.8867 \pm 0.1684$ & & \\
\hline
\end{tabular}

SI: Stability index. ; $\mathrm{X}^{-}$: Mean; SD: Standard deviation; P-value:

Level of Significance, ${ }^{*} p<0.05$ : Significant

mance of most functional skills, helping children to recover from unexpected balance disturbances, either due to slips and trips or to self-induced instability when executing any movement that brings them toward the edge of their limits of stability [39].

The pre-treatment mean values of overall stability index, anteroposterior stability index, and mediolateral stability index of the dynamic balance test for both groups, showed an increase in their values, which indicates that those children had balance problems. These results were consistent with those who reported that higher stability index was due to poor standing stability [40]. Also the pre-treatment mean values of this study are in agreement with the findings of Roncesvalles et al., [41], who stated that one of the contributing factors in stability of children with spastic diplegia is a poor ability to increase muscle response amplitude when balance threats increase in magnitude.

Previous studies had reported that children with spastic hemiplegia show problems with the timing of muscle responses. Such problems include delayed onset of contraction in the ankle muscle and co-contraction of agonist and antagonist muscles at a joint. Such changes in neuromuscular response characteristics would lead to a slower and less organized recovery of balance [42].

The results are supported by a study of Rasti et al. [43] which revealed that $\mathrm{KT}$ is very effective intervention in children with spastic CP children who were classified in level I and II in the gross motor functional classification system and all of them showing improvement in their gross motor abilities.

Improvement reported regarded to KT groups may be attributed to the effects of $K T$ in rehabilitation of children with $C P$, this come in agreement with Da Costa et al., [5]. who confirmed that KT for the tibialis anterior and quadriceps muscles has a benefit effect in the enhancement of static and dynamic balance also functional activities in children with cerebral palsy.

The obtained results of this study were also agreed with Morris et al., [44] who confirmed that improvement in balance of children in KT group may be attributed to the effects of taping on muscular facilitation or inhabitation, the re-alignment of joints, improving proprioception and postural support in children with $\mathrm{CP}$.
The significant improvement in post treatment mean values of balance indices in hemiparetic group whom underwent $\mathrm{KT}$ treatment of the affected ankle may be explained by the influence of taping technique on realignment of the ankle joint in mid position which enhance proper alignment with the least expenditure of muscle energy and postural tone, in addition of the proprioceptive influence in the form of cutaneous stimulation that enhance hemiparetic children awareness to maintain his/her ankle and foot in an upright mid position that means effective postural control and body alignment. This explanation was supported by the work of Spanos et al,. [45] who studied the effects of taping on the proprioception of the ankle.

On the other hand, our results disagreed with Ekiz el al., [46] who showed no functional effect of the KT application on stroke patients. Also, another study stated that there was no direct effect of KT application on the gross motor function [47].

Effects of ES on improving the stability indices of the CP children were supported by the study of Schuhfried et al., [48] who assessed the impact of ES on treatment of central nervous system lesions that improve motor control, reduce spasticity, prevent deformities and generally used to improve the function of the affected extremity and also with Hamid and Hayek [49] who investigated the effectiveness of NMES as a safe method that stimulates the injured neuromuscular system in an attempt to reacquire or improve function with CP children.

The improvement of stability indices which is reported in the spastic hemiparetic cerebral palsied children who received ES, may be attributed to its effect on dorsiflexors as the imbalance at the ankle joint caused by a spastic calf muscle inhibits the development of the ankle dorsiflexors strength, making them weak and elongated [50]. Neuromuscular electrical stimulation is commonly used to decrease muscle spasticity and to strengthen the weakened antagonist muscles. Typically, NMES is applied to antagonist muscles (ankle dorsiflexor) to achieve an antispastic effect on agonist muscles (triceps surae) by the reciprocal inhibition [51] NMES contributes to improving joint range of motion. Hazelwood et al., [52] found an increase in passive range of movement at the ankle and ankle dorsiflexion strength in children with hemiplegic cerebral palsy after electrical stimulation of the anterior tibial muscles. This is affecting the ability to respond to the perturbations and improves balance control of the ankle.

The balance improvement in diplegic children who undergone ES may be explained by the work of Daichman et al., [53] who reported the effects of using NMES on impairments and functional skills of a child with spastic diplegic CP. They concluded that NMES may allow a child with poor motor control to participate in a progressive strength-training program. NMES may also lead to motor learning.

The obtained results of this study were also agreed with Wright et al., [54] who reported that use NMES on tibialis anterior muscle is based on NMES's potential to strengthen 
Zaky et al, Physical Therapy and Rehabilitation 2019,

http://www.hoajonline.com/journals/pdf/2055-2386-6-2.pdf

this weak muscle, allow reciprocal inhibition of spastic gastrocnemius and also produce a stretch reflex. Furthermore, as a result of motor relearning, these changes may be maintained after the treatment period.

Concerning the physical therapy program received by children in both groups, a study confirmed that a rehabilitation program gives a benefit for CP children and help in their progress [55], this comes in accordance with the results of the current study.

The effect of the exercise program also supported by the work of Eek et al., [56] who showed that strengthening programs have a positive effect on balance and gait via improving muscle strength, enhancing plantar-flexor generating power at push off, increasing the ability to balance on one leg, that may improve balance from standing and promote independence in children with CP.

The present study had some limitations. Sample size was small, and there was no control group for both interventions , also lack of long-term follow up. Future studies are needed to report the sustainability of the effect of KT and ES on balance of $\mathrm{CP}$ children.

\section{Conclusion}

This study demonstrated the effectiveness of using two rehabilitation therapies for patients with cerebral palsy that focus on improving standing balance because postural balance is integral to all motor abilities. Therefore, improving postural balance would improve function. Among the alternatives, we propose that using $\mathrm{KT}$ treatment is more beneficial in treating diplegic $\mathrm{CP}$ children, and using $\mathrm{ES}$ is more effective in hemiparetic CP children.

\section{Competing interests}

The authors declare that they have no competing interests.

Authors' contributions

\begin{tabular}{|l|c|c|c|}
\hline Authors' contributions & NAZ & NST & MBB \\
\hline Research concept and design & $\checkmark$ & $\checkmark$ & -- \\
\hline Collection and/or assembly of data & $\checkmark$ & $\checkmark$ & -- \\
\hline Data analysis and interpretation & $\checkmark$ & $\checkmark$ & $\checkmark$ \\
\hline Writing the article & $\checkmark$ & $\checkmark$ & -- \\
\hline Critical revision of the article & $\checkmark$ & $\checkmark$ & $\checkmark$ \\
\hline Final approval of article & $\checkmark$ & $\checkmark$ & -- \\
\hline Statistical analysis & $\checkmark$ & $\checkmark$ & $\checkmark$ \\
\hline
\end{tabular}

Acknowledgment

The authors would like to express their appreciation to all children and their parents who participated in this study with cooperation.

Publication history

Editor: Mohammad H. Hadadzadeh, Wheeling Jesuit University, USA. Received: 24-Oct-2018 Final Revised: 20-Dec-2018

Accepted: 24-Dec-2018 Published: 06-Jan-2019

\section{References}

1. Hielkema T and Hadders-Algra M. Motor and cognitive outcome after specific early lesions of the brain - a systematic review. Dev Med Child
Neurol. 2016; 58 Suppl 4:46-52. | Article | PubMed

2. Jan MM. Cerebral palsy: comprehensive review and update. Ann Saudi Med. 2006; 26:123-32. | Article | PubMed Abstract | PubMed FullText

3. Nelson KB. Can we prevent cerebral palsy? N Engl J Med. 2003; 349:1765-9. | Article | PubMed

4. Christensen D, Van Naarden Braun K, Doernberg NS, Maenner MJ, Arneson CL, Durkin MS, Benedict RE, Kirby RS, Wingate MS, Fitzgerald $R$ and Yeargin-Allsopp M. Prevalence of cerebral palsy, co-occurring autism spectrum disorders, and motor functioning - Autism and Developmental Disabilities Monitoring Network, USA, 2008. Dev Med Child Neurol. 2014; 56:59-65. | Article | PubMed Abstract | PubMed FullText

5. da Costa CS, Rodrigues FS, Leal FM and Rocha NA. Pilot study: Investigating the effects of Kinesio Taping(R) on functional activities in children with cerebral palsy. Dev Neurorehabil. 2013; 16:121-8. | Article I PubMed

6. Aboutorabi A, Arazpour M, Ahmadi Bani M, Saeedi H and Head JS. Efficacy of ankle foot orthoses types on walking in children with cerebral palsy: A systematic review. Ann Phys Rehabil Med. 2017; 60:393-402. I Article | PubMed

7. Sanger T.D. Movement Disorders in Cerebral Palsy. J Pediatr Neurol. 2015; 13:198-207.

8. Wright M and Wallman L. Cerebral Palsy. In: Campbell S.K., Palisano R.J., Orlin M.N.: Physical therapy for children. 4th ed. Missouri: ElsevierSanders. 2012; 577-627.

9. Shamsoddini A, Amirsalari S, Hollisaz MT, Rahimnia A and Khatibi-Aghda A. Management of spasticity in children with cerebral palsy. Iran J Pediatr. 2014; 24:345-51. | PubMed Abstract | PubMed FullText

10. Shamsoddini A. Comparison between the effect of neurodevelopmental treatment and sensory integration therapy on gross motor function in children with cerebral palsy. Iran J Child Neurol. 2010; 4:31-8. | Article

11. Novak I, McIntyre S, Morgan C, Campbell L, Dark L, Morton N, Stumbles $E$, Wilson SA and Goldsmith S. A systematic review of interventions for children with cerebral palsy: state of the evidence. Dev Med Child Neurol. 2013; 55:885-910. | Article | PubMed

12. Liao HF and Hwang AW. Relations of balance function and gross motor ability for children with cerebral palsy. Percept Mot Skills. 2003; 96:1173-84. | Article | PubMed

13. Dobson F, Morris ME, Baker R, Wolfe R and Graham H. Clinician agreement on gait pattern ratings in children with spastic hemiplegia. Dev Med Child Neurol. 2006; 48:429-35. | Article | PubMed

14. Patterson KK, Gage WH, Brooks D, Black SE and Mcllroy WE. Evaluation of gait symmetry after stroke: a comparison of current methods and recommendations for standardization. Gait Posture. 2010; 31:241-6. | Article I PubMed

15. Basu AP, Pearse J, Kelly S, Wisher V and Kisler J. Early intervention to improve hand function in hemiplegic cerebral palsy. Front Neurol. 2014; 5:281. | Article | PubMed Abstract | PubMed FullText

16. Das S.P, Singh D, Sahoo J, Prasad S.V, Mohanty R.N and Das S.K. Lower limb alignment in cerebral palsy. Indian J Orthop. 2005; 39:248-53. I Article

17. Kelle1 B, Tas D and Kozanoğlu E. Kinesio taping application in a pediatric patient with spinal muscular atrophy. Cukurova Med J. 2016; 41:386389. | Article

18. Cepeda J., Fishweicher A., Gleeson M., Greenwood S and Motyka-Miller C. Does Kinesio Taping of the abdominal muscles improve the supineto-sit transition in children with hypotonia. 2008;12:2011. | Pdf

19. Chae J, Fang ZP, Walker M, Pourmehdi S and Knutson J. Intramuscular electromyographically controlled neuromuscular electrical stimulation for ankle dorsiflexion recovery in chronic hemiplegia. Am J Phys Med Rehabil. 2001; 80:842-7. | Article | PubMed

20. Damiano DL, Dodd K and Taylor NF. Should we be testing and training muscle strength in cerebral palsy? Dev Med Child Neurol. 2002; 44:6872. | Article | PubMed

21. Wiley ME and Damiano DL. Lower-extremity strength profiles in spastic cerebral palsy. Dev Med Child Neurol. 1998; 40:100-7. | Article | PubMed 
22. Lephart SM, Pincivero $D$ and Henry. Learning effects and reliability of the biodex stability system. (C) BIODEX MEDICAL SYSTEMS, INC.

23. Hinman M.R. Factors affecting reliability of the Biodex Balance System : a summary of four studies. J Sport Rehabil. 2000; 9:240-252. | Article

24. Palisano R, Rosenbaum P, Walter S, Russell D, Wood E and Galuppi B. Development and reliability of a system to classify gross motor function in children with cerebral palsy. Dev Med Child Neurol. 1997; 39:214-23. | Article | PubMed

25. Bohannon RW and Smith MB. Interrater reliability of a modified Ashworth scale of muscle spasticity. Phys Ther. 1987; 67:206-7. | PubMed

26. Rozzi S., Lephart $S$ and Sterner R et al. Balance training for persons with functionally unstable ankles. In: Rusnak-Smith S., Rosen E., Moffat M. :Musculoskeletal essentials: applying the preferred physical therapist practice patterns. $2^{\text {nd }}$ ed: SLACK Incorporated. 2006; 350.

27. Kase K, Wallis J and Kase T. Clinical therapeutic applications of the Kinesio taping method. $2^{\text {nd }}$ ed. Tokyo: Ken Ikai; 2003.

28. Kahanov L. Kinesio taping: An overview of use with athletes, part II. Ath Ther Today. 2007; 12:5-7. I Article

29. Shokr A.M, Elmeniawy G.H, Amer H.A and Thabet N S. Sitting Control in Response to Electrical Stimulation in Spastic Diplegic Cerebral Palsied Children. Master Thesis, Faculty of Physical Therapy, Cairo University. 2013.

30. Finn, et al, Southern Connecticut State Univ. Stability Performance Assessment Among Subject of Disparate Balance Abilities. 1999.

31. Ibrahim A.F, Abd El-Azim F. H and Elmonem A.M. Correlation between kinematic gait parameters and balance in children with diplegic cerebral palsy. Master thesis, Faculty of Physical Therapy, Cairo University. 2015.

32. Ballard T. Product Profile: The Biodex Balance System, Biodex Medical Systems. 2011; 91-111.

33. Levitt S. Treatment of cerebral palsy and motor delay. Blackwell Scientific publication, $4^{\text {th }}$ ed Oxford. 2004; 1-30, 169-223.

34. Kenzo Kase D, Patricia M and Audry Y. Kinesio Taping in Pediatrics Fundamentals and Whole Body Taping. Kinesio 2006.

35. Ragab R, Elmeniawy G, El-barbary $\mathrm{H}$ and Husein Z. Role of ankle Taping on balance in children with hemiparetic Cerebral Palsy. Master thesis, Faculty of Physical Therapy, Cairo University. 2013.

36. Kerr C, McDowell B and McDonough S. Electrical stimulation in cerebral palsy: a review of effects on strength and motor function. Dev Med Child Neurol. 2004; 46:205-13. | Article | PubMed

37. Ferrari A, Tersi L, Sghedoni A and Chiari L. Functional reaching discloses perceptive impairment in diplegic children with cerebral palsy. Gait Posture. 2010; 32:253-8. | Article | PubMed

38. Bodkin AW, Robinson C and Perales FP. Reliability and validity of the gross motor function classification system for cerebral palsy. Pediatr Phys Ther. 2003; 15:247-52. | Article | PubMed

39. Woollacott $\mathrm{MH}$ and Shumway-Cook A. Postural dysfunction during standing and walking in children with cerebral palsy: what are the underlying problems and what new therapies might improve balance? Neural Plast. 2005; 12:211-9; discussion 263-72. | Article I PubMed Abstract | PubMed FullText

40. Paterno MV, Myer GD, Ford KR and Hewett TE. Neuromuscular training improves single-limb stability in young female athletes. J Orthop Sports Phys Ther. 2004; 34:305-16. | Article | PubMed

41. Roncesvalles MN, Woollacott MW and Burtner PA. Neural factors underlying reduced postural adaptability in children with cerebral palsy. Neuroreport. 2002; 13:2407-10. | Article | PubMed

42. Batshaw $M$ and Perret Y. Children with disabilities: A Medical primer 2005, $3^{\text {rd }}$ ed.Tornoto. 2005.

43. Shamsoddini A, Rasti Z, Kalantari M, Hollisaz MT, Sobhani V, Dalvand H and Bakhshandeh-Bali MK. The impact of Kinesio taping technique on children with cerebral palsy. Iran J Neurol. 2016; 15:219-227. | PubMed Abstract | PubMed FullText

44. Morris D, Jones D, Ryan $H$ and Ryan CG. The clinical effects of Kinesio(R) Tex taping: A systematic review. Physiother Theory Pract. 2013; 29:259-
70. | Article | PubMed

45. Spones S, Brunswi C.M and Billis E. The effect of prorioception of the ankle in non-weight bearing position amongst atheletes. J Foot. 2008; 18:25-33. | Article

46. Ekiz T, Aslan MD and Ozgirgin N. Effects of Kinesio Tape application to quadriceps muscles on isokinetic muscle strength, gait, and functional parameters in patients with stroke. J Rehabil Res Dev. 2015; 52:323-31. | Article | PubMed

47. Simsek TT, Turkucuoglu B, Cokal N, Ustunbas G and Simsek IE. The effects of Kinesio(R) taping on sitting posture, functional independence and gross motor function in children with cerebral palsy. Disabil Rehabil. 2011; 33:2058-63. | Article | PubMed

48. Schuhfried O, Crevenna R, Fialka-Moser V and Paternostro-Sluga T. Noninvasive neuromuscular electrical stimulation in patients with central nervous system lesions: an educational review. J Rehabil Med. 2012; 44:99-105. | Article | PubMed

49. Hamid S and Hayek R. Role of electrical stimulation for rehabilitation and regeneration after spinal cord injury: an overview. Eur Spine J. 2008; 17:1256-69. | Article | PubMed Abstract | PubMed FullText

50. Renshaw T.S, Green N.E and Griffin P.P et al. Cerebral palsy: orthopaedic management. J Bone Joint Surg. 1995; 77A:1590-606.

51. Seib TP, Price R, Reyes MR and Lehmann JF. The quantitative measurement of spasticity: effect of cutaneous electrical stimulation. Arch Phys Med Rehabil. 1994; 75:746-50. I Article I PubMed

52. Hazlewood ME, Brown JK, Rowe PJ and Salter PM. The use of therapeutic electrical stimulation in the treatment of hemiplegic cerebral palsy. Dev Med Child Neurol. 1994; 36:661-73. I Article I PubMed

53. Daichman J, Johnston TE, Evans $K$ and Tecklin JS. The effects of a neuromuscular electrical stimulation home program on impairments and functional skills of a child with spastic diplegic cerebral palsy: a case report. Pediatr Phys Ther. 2003; 15:153-8. | Article I PubMed

54. Wright PA, Durham S, Ewins DJ and Swain ID. Neuromuscular electrical stimulation for children with cerebral palsy: a review. Arch Dis Child. 2012; 97:364-71. | Article | PubMed

55. Icagasioglu A, Mesci E, Yumusakhuylu Y, Turgut ST and Murat S. Rehabilitation outcomes in children with cerebral palsy during a $\mathbf{2}$ year period. J Phys Ther Sci. 2015; 27:3211-4. | Article | PubMed Abstract | PubMed FullText

56. Eek MN, Tranberg R, Zugner R, Alkema K and Beckung E. Muscle strength training to improve gait function in children with cerebral palsy. Dev Med Child Neurol. 2008; 50:759-64. | Article I PubMed

Citation:

Zaky NA, Thabet NS and Banoub MB. Role of two therapeutic interventions on balance in children with spastic diplegia and hemiparasis : A comparative study. Phys Ther Rehabil. 2019; 6:2. http://dx.doi.org/10.7243/2055-2386-6-2 